even before we do, the GRP system is likely to become a focus for research into new, selective, anxiety-relieving drugs. Yadin Dudai is in the Department of Neurobiology, Weizmann Institute of Science, Rehovot 76100, Israel. e-mail:yadin.dudai@weizmann.ac.il

1. LeDoux, J. E. The Emotional Brain: The Mysterious Underpinning of Emotional Life (Simon \& Schuster, New York, 1996).

2. Shumyatsky, G. P. et al. Cell 111, 905-918 (2002).

3. Aggleton, J. P. (ed.) The Amygdala: A Functional Analysis
(Oxford Univ. Press, Oxford, 2000)

4. Cahill, L., Weinberger, N. M., Roozendaal, B. \& McGaugh, J. L. Neuron 23, 227-228 (1999).

5. Maren, S., Aharonov, G., Stote, D. L. \& Fanselow, M. S. Behav. Neurosci. 110, 1365-1374 (1996).

6. Lamprecht, R., Farb, C. R. \& LeDoux, J. E. Neuron 36, 727-738 (2002)

7. Ressler, K. J., Paschall, G., Zhou, X. L. \& Davis, M. J. Neurosci. 22, 7892-7902 (2002)

8. Rogan, M. T., Staubli, U. V. \& LeDoux, J. E. Nature 390, 604-607 (1997).

\title{
Thermal physics
}

\section{Heat in one dimension}

\author{
Roberto Livi and Stefano Lepri
}

Heat is transferred along a temperature gradient, from hot to cold, at a rate determined by the thermal conductivity of the material. But is the situation so straightforward in fewer than three dimensions?

$\mathrm{M}$ any phenomena in nature occur as the result of some kind of imbalance. For instance, an electric current flows when there is a difference in electric potential along a conductor (such as when an electric field is applied), and heat is transported when there is a temperature gradient between two boundaries of a material. Despite their ubiquity in everyday life, many aspects of such phenomena are still the subject of debate among theoretical physicists. One central issue is the role of spatial constraints, caused by the dimensionality of a system: the response of a system to external forces is intimately related to statistical fluctuations within it, and these, in turn, depend strongly on whether the system is one-, twoor three-dimensional. What happens to energy or charge transport in systems that are effectively one-dimensional, such as a nanowire or a DNA molecule? Onuttom Narayan and Sriram Ramaswamy ${ }^{1}$ give their answer in Physical Review Letters.

Because of the variety and complexity of specific interactions, simplified microscopic
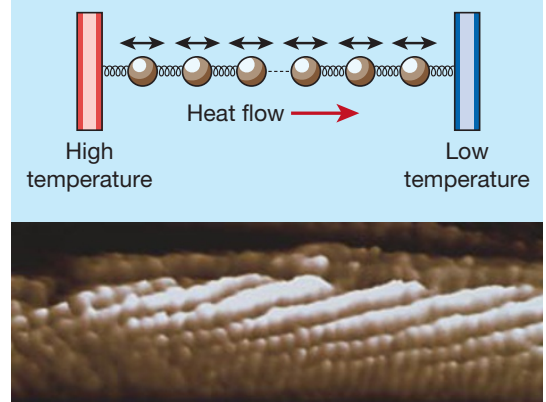

Figure 1 Simple models and real materials. Modelling anomalous heat conduction in one-dimensional systems of interacting particles — such as a chain of oscillating particles that connect two reservoirs at different temperatures (above) - is relevant for understanding heat transport in single-walled carbon nanotubes (below). models are an invaluable tool for the study of transport mechanisms in reduced dimensions (Fig. 1). An example is the old problem of heat conduction. Our own simulations ${ }^{2,3}$ using models of classical low-dimensional fluids and crystals show that the thermal conductivity should increase with the system size. In other words, the larger the system, the more efficiently heat is transported (assuming that the density of the material and the temperature gradient are fixed) — in physical terms, the mean free path of the 'heat carriers' increases with the length of the sample.

Narayan and Ramaswamy ${ }^{1}$ are now able to justify this unusual behaviour. They considered a normal fluid, in which the only quantities that vary in time and space are mass, momentum and energy density. They show analytically that the usual concept of thermal conductivity is not well defined for a system of fewer than three dimensions confirming that space dimensionality is crucial in anomalous transport properties. More specifically, they have estimated that the thermal conductivity diverges as the length of a one-dimensional system increases, following a power law with exponent $1 / 3$; for a two-dimensional system, the divergence is much weaker and logarithmic. But such anomalous behaviour disappears in three dimensions.

Transport anomalies such as this have been found in many microscopic models, including one-dimensional crystals. Although it may seem strange to consider a one-dimensional crystal as a fluid, it is nonetheless well known that mechanical vibrations in a crystal structure can be described by hydrodynamic equations similar to those used in a fluid, as interacting phonons in a crystal behave similarly to particles in a fluid. And as Narayan and Ramaswamy ${ }^{1}$ discuss, it is reasonable to expect that the same equations should also be applicable to models of low-dimensionallattices.

For the case of particular lattices of parti- cles known as Fermi-Pasta-Ulam chains, we have performed extensive computer simulations $^{3}$ that also indicate a power-law divergence for the thermal conductivity in one-dimensional systems. But our finding for the index of the power law (a value greater than 0.36) is not fully in agreement with Narayan and Ramaswamy's prediction of $1 / 3$. These authors suggest that the discrepancy might arise because, in the one-dimensional case, a fluid and a lattice shouldn't be considered to be exactly equivalent, at least for the intermediate sizes and timescales currently accessible in simulations. In this respect, there are open questions: can the anomalous behaviour actually be described by universal scaling laws, and to what extent does it depend on the nature of the microscopic interactions?

The conceptual challenge is not the only reason for studying energy transport in spatially constrained systems - there is also a variety of real systems in which these anomalies are important. Anisotropic crystals, magnetic chains, polymers and semiconductor films or wires are all examples of systems in which modern experimental techniques can probe the transport properties directly. Single-walled nanotubes (Fig. 1) are known through experiment to have an unusually high thermal conductivity, which is attributed mainly to quasi-one-dimensional lattice vibrations $s^{4,5}$, and it is reasonable to expect that the scaling laws derived for simple models should apply to nanotubes as well ${ }^{6}$. Although, so far, an experimental test is lacking, molecular-dynamics simulations that use realistic energy potentials for the carbon atoms support this idea ${ }^{7}$. If the thermal conductivity did increase with nanotube length in a well-defined way, this would be a very promising feature to use in technological applications, such as the design of components that dissipate heat efficiently in nanocircuits.

In building models of energy-transport processes, the aim is to single out generic physical features, although this might sometimes be at the price of drastic simplifications. Hopefully, in the end the results will go beyond pure academic interest and suggest new ideas for technological applications perhaps the reader is astonished that so many interesting and innovative ideas are still emerging from classical mechanics.

Roberto Livi is in the Dipartimento di Fisica, and INFM and INFN, and Stefano Lepri is in the

Dipartimento di Energetica and INFM, Università

di Firenze, Florence, Italy.

e-mails: livi@fi.infn.it

stefano.lepri@unifi.it

1. Narayan, O. \& Ramaswamy, S. Phys. Rev. Lett. 89, 200601 (2002).

2. Lepri, S., Livi, R. \& Politi, A. Phys. Rev. Lett. 78, 1896-1899 (1997).

3. Lepri, S., Livi, R. \& Politi, A. Phys. Rep. (in the press); Preprint cond-mat/0112193 (2001), http://arXiv.org

4. Hone, J. et al. Phys. Rev. B 59, R2514-R2516 (1999).

5. Kim, P. et al. Phys. Rev. Lett. 87, 215502 (2001).

6. Grassberger, P. \& Yang, L. Preprint cond-mat/0204247 (2002); http://arXiv.org

7. Maruyama, S. Physica B 323, 193-195 (2002). 